\title{
Deep or Superficial erector spinae block: A pilot study to compare the efficacy of two techniques
}

\author{
Dr. Chandni Sinha, Dr. Ajeet Kumar, Dr. Amarjeet Kumar, Dr Poonam Kumari \\ Department of Anaesthesia, AlIMS, Patna India
}

\section{Introduction}

- Patients undergoing breast cancer surgeries face significant post-operative pain.

- Achieving adequate perioperative analgesia can be challenging

\section{Method}

-Prospective randomized double blind study.

- Institutional ethics Committee clearance and CTRI registration (CTRI/2019/01/017349).

-The study done over a period of 6 months, between January 2019 to June 2019.

-Forty female patients, age group 18 to 60 years undergoing unilateral Modified Radical Mastectomy.

-ASA I/II, no history of psychiatric disorder, local anesthetic allergy, not on any chronic analgesic therapy.

-Group I received ESP block. (Deep): $25 \mathrm{ml}$ of $0.2 \%$ Ropivicaine

-Group II received ESP block. (Superficial) $25 \mathrm{ml}$ of $0.2 \%$ Ropivicaine

-The blocks were performed under all aseptic precautions around 40 mins before surgery General Anaesthesia was administered in standardised manner.

-Postoperatively, all patients received iv morphine Patient Controlled Analgesia.

\section{Assessment}

-The duration of analgesia (time to first rescue analgesia after administration of block)

- Total analgesic consumption in $24 \mathrm{~h}$ after surgery.

-Sensory blockade (30 minutes after the block)

Result

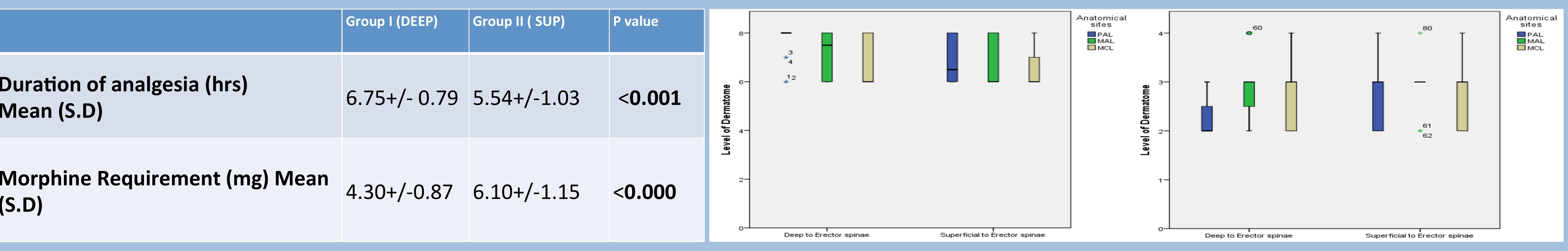

Discussion

- ESP block was introduced by Forero et al who used this simple interfascial plane block in cases of severe neuropathic pain post trauma/ malignancy/thoracotomy. [1]

- Erector Spinae block has been used for VATS, thoracotomy, breast surgery, abdominal bariatric surgery, ventral hernia repair $[2,3,4]$

\section{Limitation}

- Post mastectomy chronic pain was not assessed

\section{Conclusion}

- Deep to ESP muscle is a more effective technique than the superficial one, in terms of analgesia and opioid requirement.

\section{References}

1.Forero M, Adhikary SD, Lopez H, Tsui C,Chin kjThe Erector Spinae Plane Block: A Novel Analgesic Technique in Thoracic Neuropathic Pain

2.Ohgoshi $\mathrm{Y}$, Ikeda T, Kurahashi K. Continuous erector spinae plane block provides effective perioperative analgesia for breast reconstruction using tissue expanders: a report of two cases. J Clin Anesth. 2018; 44:1-2.

3.Chin KJ, Malhas L, Perlas A. The erector spinae plane block provides visceral abdominal analgesia in bariatric surgery: a report of 3 cases. Reg Anesth Pain Med. 2017; 42:372-376. 